\title{
Harmonics generation in electron-ion collisions in a short laser pulse
}

\author{
H. Haberland \\ Institut für Physik, Universität Greifswald, Domstr. 10a, 17487 Greifswald \\ M. Bonitz and D. Kremp \\ Fachbereich Physik, Universität Rostock \\ Universitätsplatz 3, D-18051 Rostock, Germany
}

(July 4, 2021)

Anomalously high generation efficiency of coherent higher field-harmonics in collisions between oppositely charged particles in the field of femtosecond lasers is predicted. This is based on rigorous numerical solutions of a quantum kinetic equation for dense laser plasmas which overcomes limitations of previous investigations.

Modern high intensity short-pulse lasers [1] have made it possible to create dense correlated plasmas under laboratory conditions promising a large variety of applications, e.g. 22,33. Remarkable progress has been made in the field of time-resolved plasma diagnostics [4], interesting high-field phenomena have been observed, including production of multi-keV electrons and the generation of higher field harmonics (bremsstrahlung, BS) in neutral gases. On the other hand, despite early predictions [5], harmonics generation in an ionized medium due to collisions of oppositely charged particles in the field has not been observed experimentally [6]. The reason is the low electron-ion collision frequency $\nu_{e i}$ in a low density equilibrium plasma [7]. In this Letter we demonstrate a drastic, more than 6 orders of magnitude, efficiency increase for dense nonequilibrium plasmas excited by a femtosecond laser pulse which should make this fundamental effect observable experimentally. Moreover, we predict coherent harmonics pulses which may be significantly shorter than the pump pulse.

A full theoretical description of dense laser plasmas requires the selfconsistent treatment of i) hydrodynamic mass and energy transport of electrons and heavy particles, ii) collective (mean-field) phenomena and iii) elastic and inelastic carrier collisions in the presence of the field. In recent years there have been substantial advances in the theoretical description of the first two effects, see e.g. [3,8,9] and [10 12], respectively. However, these treatments, usually being based on Vlasov, Fokker-Planck or particle in cell simulations, either neglect collisions completely or study them in a strongly simplified manner. In particular, they neglect the field effect on the collision process. This may be justified for very strong fields where the electron oscillation velocity $v_{0} \equiv e E_{0} /\left(m_{e} \Omega\right)$ is much larger than the thermal velocity $v_{t h}$, and the collision fre- quencies $\nu_{e e}$ and $\nu_{e i}$ are much below the oscillation frequency $\Omega$ of the field. However, for field intensities below $\left.10^{15} \mathrm{~W} / \mathrm{cm}^{2}\right)$ and/or high plasma density $\left(n>10^{20} \mathrm{~cm}^{-3}\right)$ and low temperature, collisions are the dominant factor in field-matter interaction at short times. At a later stage, due to hydrodynamic motion of electrons and ions, plasma heating and expansion, field effects will govern the plasma behavior, although collisional processes such as inverse bremsstrahlung, e.g. 13,.5.9. or impact ionization/recombination remain important.

When this transition occurs and what physical processes take place at the early stage is not known until now - to answer these questions is the main goal of this paper. Our analysis reveals that during this initial stage the plasma has a strongly anisotropic and nonMaxwellian electron distribution function which develops bunches of fast electrons. As a result of electron-electron collisions, eventually a monotonically decaying distribution is approached, but this thermalization takes about $t_{\text {rel }} \sim 50 \ldots 100 \mathrm{fs}$. Finally, the most interesting effect is that, under the strong nonequilibrium conditions at these short times, the generation of higher field harmonics in e-i collisions is strongly enhanced.

The analysis of the initial stage of laser-matter interaction requires a quantum kinetic equation which fully includes nonideal plasma effects the importance of which has been noted frequently, e.g. [8, 12]. Such an equation has recently been derived 114 and has the form

$$
\left\{\frac{\partial}{\partial t}+e_{a} \mathbf{E}(t) \nabla_{\mathbf{k}}\right\} f_{a}\left(\mathbf{k}_{a}, t\right)=\sum_{b} I_{a b}\left(\mathbf{k}_{a}, t\right)
$$

where for the initial period spatial homogeneity may be assumed. The collision integrals are given by

$$
\begin{array}{r}
I_{a b}\left(\mathbf{k}_{a}, t\right)=2 \int \frac{d \mathbf{k}_{b} d \overline{\mathbf{k}}_{a} d \overline{\mathbf{k}}_{b}}{(2 \pi \hbar)^{6}}\left|V_{a b}(\mathbf{q})\right|^{2} \delta\left(\mathbf{k}_{a b}-\overline{\mathbf{k}}_{a b}\right) \\
\times \int_{t_{0}}^{t} d \bar{t} \cos \left[\frac{\epsilon_{a b}-\bar{\epsilon}_{a b}}{\hbar}(t-\bar{t})-\frac{\mathbf{q}}{\hbar} \mathbf{R}_{a b}(t, \bar{t})\right] \\
\times\left.\left\{\bar{f}_{a} \bar{f}_{b}\left[1-f_{a}\right]\left[1-f_{b}\right]-f_{a} f_{b}\left[1-\bar{f}_{a}\right]\left[1-\bar{f}_{b}\right]\right\}\right|_{\bar{t}},
\end{array}
$$

where $a, b$ label electrons and ions, and we denoted $\epsilon_{a b} \equiv$ $\epsilon_{a}+\epsilon_{b}, \epsilon_{a} \equiv p_{a}^{2} / 2 m_{a}, \mathbf{k}_{a b} \equiv \mathbf{k}_{a}+\mathbf{k}_{b}$, and $\mathbf{q} \equiv \mathbf{k}_{a}-\overline{\mathbf{k}}_{a}$. $V_{a b}$ is the Fourier transform of the screened Coulomb potential $V_{a b}(q)=4 \pi e_{a} e_{b} /\left[q^{2}+\kappa(t)^{2}\right],[\kappa(t)$ is the inverse nonequilibrium screening length]. This allows to 
avoid any cutoff (Coulomb logarithm). We underline that the collision integral $I_{a b}$ is exact (within the static weakcoupling limit). In particular, it permits a rigorous investigation of the short-time physics and, for long times $t \gg 2 \pi / \omega_{p l}\left[\omega_{p l}=\left(4 \pi n e^{2} / m\right)^{1 / 2}\right.$ is the plasma frequency] and zero field $\left(R_{a b}=0\right)$, it goes over to the familiar Landau collision integral $\int d \bar{t} \cos \ldots \rightarrow \hbar \pi \delta\left(\epsilon_{a b}-\bar{\epsilon}_{a b}\right)$. Moreover, this integral includes Pauli blocking (spin statistics) and it conserves the total energy (kinetic plus potential energy) which is important for dense nonideal plasmas.

Most importantly, the integral $I_{a b}$ includes the influence of a strong field on the collision process of two particles of species $a$ and $b$ exactly, as well as the effect of a finite collision duration $\tau_{\text {coll }}=t_{2}-t_{1}$ which is crucial for the results shown below. Indeed, during the time $\tau_{\text {coll }}$ each of the colliding particles gains a certain momentum $\mathbf{Q}_{a}\left(t_{1}, t_{2}\right)$ in the field, and a pair of colliding (oppositely charged) particles is displaced by the field a distance $R_{a b}\left(t_{1}, t_{2}\right)$. For a harmonic field, $\mathbf{E}(t)=\mathbf{E}_{0} \cos \Omega t$,

$$
\begin{aligned}
\mathbf{Q}_{a}\left(t_{1}, t_{2}\right) & =\frac{-e_{a} \mathbf{E}_{0}}{\Omega}\left(\sin \Omega t_{1}-\sin \Omega t_{2}\right), \\
\mathbf{R}_{a b}\left(t_{1}, t_{2}\right) & =\left(\frac{e_{a}}{m_{a}}-\frac{e_{b}}{m_{b}}\right) \frac{\mathbf{E}_{0}}{\Omega^{2}} \\
& \times\left\{\cos \Omega t_{1}-\cos \Omega t_{2}-\Omega\left(t_{2}-t_{1}\right) \sin \Omega t_{1}\right\},
\end{aligned}
$$

and these effects are growing with increasing field strength. The momentum shift enters the arguments of the distribution functions in the collision integral (2), (time-dependent intra-collisional field effect): $f_{a} \equiv$ $f\left[\mathbf{k}_{a}+\mathbf{Q}_{a}(t, \bar{t}), \bar{t}\right] ; \bar{f}_{a} \equiv f\left[\overline{\mathbf{k}}_{a}+\mathbf{Q}_{a}(t, \bar{t}), \bar{t}\right]$, whereas $\mathbf{R}_{a b}$ modifies the energy balance of the two-particle collisions in a monochromatic field. Thus the collision integral (2) selfconsistently includes nonlinear field effects, such as harmonics generation, emission (absorption) of laser photons during the two-particle scattering [(inverse) BS] and generalizes previous treatments of these phenomena, e.g. [5,9], to the case of arbitrary short times, nonequilibrium distributions and modifications by dense plasma effects.

We expect the most interesting physics to occur in situations where the effects of collisions and of the field are of the same order: (i) when the field frequency $\Omega$ is in the range of the collision frequency $\nu_{e i}$ and the plasma frequency $\omega_{p l}$ and, (ii) when the field induced particle velocities $v_{0}$ are of the same order as the thermal velocity $v_{t h}$. Thus, considering as an example a dense fully ionized hydrogen plasma in a monochromatic optical field of intermediate wavelength $\lambda=500 \mathrm{~nm}\left(\Omega=3.77 \mathrm{fs}^{-1}\right)$, the following parameter range is of interest for the simulations: densities $10^{20} \mathrm{~cm}^{-3} \leq n \leq 10^{24} \mathrm{~cm}^{-3}$, corresponding to a plasma frequency range $0.56 \mathrm{fs}^{-1} \leq \omega_{p l} \leq 56 \mathrm{fs}^{-1}$, and an initial plasma temperature of $20,000 \mathrm{~K}$ which may increase up to almost $10^{6} \mathrm{~K}$ within the first $50 \mathrm{fs}$ of the relaxation. Correspondingly, for this case field strengths of interest are $10^{7} \mathrm{~V} / \mathrm{cm} \geq E_{0} \geq 10^{9} \mathrm{~V} / \mathrm{cm}$. For stronger fields $\left(I>10^{15} \mathrm{~W} / \mathrm{cm}^{-2}\right)$ collisions will already be of minor importance.
We solve equations (1- -4 ) by direct numerical integration starting from a pre-excited electron-ion plasma in equilibrium, and the relaxation is computed over about 50 field cycles. We underline that we solve equations (1) (1) without any simplifying assumptions. In particular, momentum anisotropy of the electron distribution function as well as time and momentum retardation are fully included. The ion distribution was found to remain a Maxwellian during the considered time interval which allowed us to simplify the integral $I_{e i}$. To properly account for the time-dependence of the screened Coulomb potential $V(q)$, the inverse screening length $\kappa(t)$ is computed selfconsistently from the current nonequilibrium distribution functions. Solving the kinetic equation yields the time and momentum-dependent distribution function $f_{e}(\mathbf{k}, t)$ which allows us to compute all transport properties, including the mean electron kinetic energy density $\left\langle E_{\text {kin }}^{e}\right\rangle(t)=\frac{m_{e}}{2}\left\langle v_{e}^{2}\right\rangle$ and the electrical current density $\mathbf{j}(t)=\sum_{a} e_{a}\left\langle\mathbf{v}_{a}\right\rangle,[f$ is normalized to the density, and $\langle\ldots\rangle$ denotes averaging over $f(t)]$.

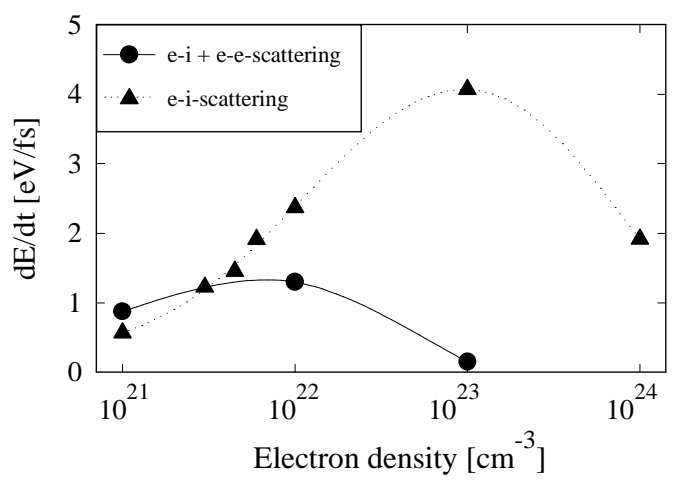

FIG. 1. Average electron kinetic energy increase versus density, with and without e-e scattering included. Field amplitude and wavelength are $E_{0}=3 \times 10^{8} \mathrm{~V} / \mathrm{cm}, \lambda=500 \mathrm{~nm}$, the initial plasma temperature is $T_{0}=20,000 \mathrm{~K}$.

Let us now come to the results. First recall that, in the collisionless case $\left(I_{a b}=0\right)$, the electron distribution would oscillate as a whole according to $\sin \Omega t$ without changing shape. This yields no net current and no energy increase (the energy oscillates with $2 \Omega$ around the ponderomotive energy $U_{\text {pond }}=m v_{0}^{2} / 4$ ). In contrast, in the presence of collisions, we observe a strong increase of the mean electron kinetic energy. After an initial transient of about $5 \mathrm{fs},\left\langle E_{\mathrm{kin}}^{e}\right\rangle$ grows almost linearly, thereby oscillating with $2 \Omega$. This energy increase is due to field-induced electron acceleration during electron-ion collisions, cf. the term $\mathbf{q R}_{e i}$ in the integral $I_{e i}$, Eq. (2). No net acceleration occurs in electron-electron collisions $\left(\mathbf{R}_{e e}=0\right)$. The energy growth rate depends on the ratio of field strength and density. This is shown in Fig. 1 
where the average slope of the energy curves is plotted for different densities. Interestingly, the most efficient plasma heating occurs around $10^{22} \mathrm{~cm}^{-3}$ which is near the critical density $n_{c r}=4.5 \cdot 10^{21} \mathrm{~cm}^{-3}$ where $\omega_{p l}=\Omega$. The maximum value of the power is about $1.5 \mathrm{eV} / \mathrm{fs}$ which roughly corresponds to the absorption of one photon per electron and laser cycle. Notice also that, although ee scattering does not contribute to the heating directly, this process may not be neglected, as this would lead to an overestimate of the power by a factor of three and a shift of the maximum to higher densities by one order of magnitude, cf. the triangles in Fig. 1.

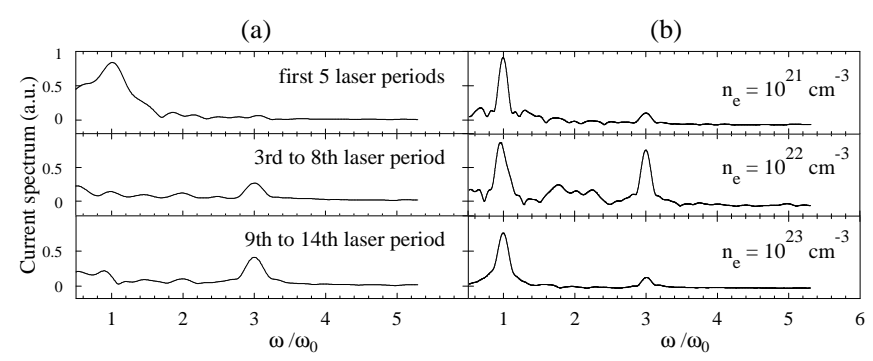

FIG. 2. Electrical current spectrum in a time-dependent electric field. The higher harmonics are the sole result of electron-ion collisions. (a) - time evolution of the spectrum for $n=10^{22} \mathrm{~cm}^{-3}$, from averaging over 5 laser periods (see text in fig.). (b) - spectrum (from averaging over the full calculation) for various densities. Other parameters are the same as in Fig. 1.

Next, we consider the electrical current density. In contrast to the collisionless case, where $\mathbf{j}_{0}(t)=e n \mathbf{v}_{0} \sin \Omega t$, collisions between electrons and ions may give rise to higher field harmonics. Indeed, electrons oscillating with the field are disturbed by the Coulomb field of ions which yields a change of the electron velocity component in field direction $\left(v_{0} \gg v_{t h}\right)$

$$
\begin{aligned}
\Delta v_{z}(t) & \approx \frac{a \sqrt{2}}{\Omega\left(1+b^{2}\right)} \frac{\sin \Omega t}{\sqrt{1+2 b^{2}+\cos 2 \Omega t}}, \\
\text { with } \quad a & =\frac{e_{e} e_{i}}{\mu r_{0}^{2}}, b=\sqrt{\frac{r_{\perp}}{r_{0}}}, r_{0}=\left(\frac{e_{e}}{m_{e}}-\frac{e_{i}}{m_{i}}\right) \frac{E_{0}}{\Omega^{2}},
\end{aligned}
$$

where $\mu^{-1}=m_{e}^{-1}+m_{i}^{-1}$, and $r_{\perp}$ is the minimal electronion distance. One readily checks that in the spectrum of (5) appear all harmonics of the field. Formula (5) indicates that the spectrum will be particularly broad for close encounters (small $r_{\perp}$, strong scattering). Further, the efficiency of harmonics generation decreases with growing field strength. On the other hand, for the case of a weak field [not covered by Eq. (5)], the harmonics yield increases with $E_{0}$ (with the number of photons), resulting in a maximum of the efficiency around $v_{0}=v_{t h}[$ [7, 15]. Quantitative estimates for the conversion efficiency $\eta_{N}$ (intensity of the $N$-th harmonics normalized to the intensity of the fundamental oscillation) can be given for the case of Maxwellian electrons. Then only odd harmonics exist and $\eta_{N}^{E Q} \sim\left(\nu_{e i} / \Omega\right)^{2} / N^{\alpha_{N}}$, where $\alpha_{N}=\alpha_{N}\left(v_{0} / v_{t h}\right)>0$ [7]. Direct evaluation of the collision integral $I_{e i}$, Eq. (2), for a dense equilibrium plasma revealed that the highest value in equilibrium is $\eta_{3}^{E Q} \sim 10^{-7}$ [15.

However, as we show now, the efficiency may be increased drastically under nonequilibrium conditions of femtosecond laser pulse excitation. Fig. 2 2 shows the results for the current spectrum obtained from the numerical solution of the quantum kinetic equation (1, 2) for $E_{0}=3 \times 10^{8} \mathrm{~V} / \mathrm{cm}$ and an initial temperature $T=20,000 \mathrm{~K}$. Fig. 2. a shows the result for a density of $n=10^{22} \mathrm{~cm}^{-3}$ at different times (obtained by Fourier transforming $\mathbf{j}(t)$ over time intervals of 5 laser periods). One clearly sees the formation of a strong third harmonics which is comparable to and may even exceed the fundamental component, i.e. $\eta_{3} \sim 1$. At later times, this peak vanishes again. We mention that higher odd harmonics are found for higher field strength also, besides, there is a clear signature of the second harmonics, cf. Fig. 2. The temporal behavior of the harmonics emission can be understood from the time evolution of the nonequilibrium cycle-averaged collision frequency

$$
\nu_{e i}\left(E_{0}, \Omega ; n, t\right)=4 \pi \frac{\Omega^{2}}{\omega_{p l}^{2}} \frac{\overline{\mathbf{j}(t) \mathbf{E}(t)}}{\overline{\mathbf{E}^{2}(t)}},
$$

which is shown in Fig. 3. Due to the laser heating of the plasma, cf. Fig. 1, and relaxation towards a Maxwellian, the collision frequency $\nu_{e i}$ decreases in time, limiting the efficiency $\eta_{N}(t)$ and, thus, the duration of the harmonics pulse.

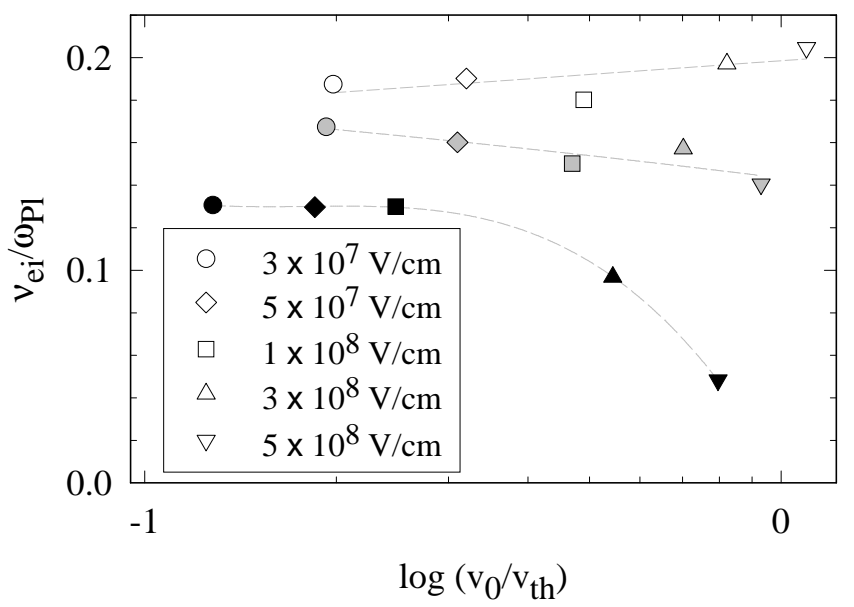

FIG. 3. Nonequilibrium collision frequency, Eq. (6), at the initial moment, after $9 \mathrm{fs}$ and after $25 \mathrm{fs}$ (white, grey and black symbols, respectively). $n=10^{22} \mathrm{~cm}^{-3}, v_{t h} \equiv \sqrt{\left\langle v_{e}^{2}\right\rangle}$. Due to collisional energy absorption, cf. Fig. 1, during the relaxation, symbols move to the left. Symbols with same shape refer to the same field strength, lines are guides to the eye.

Finally, to understand the reason for the anomalously high efficiency of harmonics generation, we consider in 
Fig. 1 the temporal evolution of the electron distribution. The three figure parts show snapshots of $f_{e}\left(k_{x}, k_{z}\right)$ after 0,6 and 12 complete field cycles. Clearly, the distribution is becoming anisotropic already during the first few laser periods. It strongly deviates from a Maxwellian, developing side peaks along the field direction, the distance of which from the origin is proportional to the field strength. This peculiar nonequilibrium behavior is caused by electron-ion collisions in the presence of the field. It is observed for all considered densities and field strengths (see above), but is strongest around $\Omega=\omega_{p l}$ and $v_{0}=v_{t h}$. Under these conditions, an electron spends the maximum possible time in the field of an ion [extending over approximately $r_{D}=\kappa^{-1}$ ] per laser cycle, $\Omega \tau_{\text {coll }} \sim \Omega r_{D} / v_{t h} \sim \Omega / \omega_{p l} \sim 1$. These are the optimal conditions for collisional e-i momentum exchange in the field, as for electron acceleration (inverse BS), cf. Fig. 1, and harmonics emission (BS), Fig. 2. b.

Our calculations show that, as a result of e-e collisions, the side peaks of $f_{e}$ start to smear out after $30 \ldots 50 \mathrm{fs}$, and after $t_{\mathrm{rel}} \sim 50 \ldots 100 \mathrm{fs}$ (depending on the density) a monotonically decaying distribution with a temperature of the order of several $10^{6} \mathrm{~K}$ is reached. By this time the plasma has become essentially collisionless: $\nu_{e i}$ has decayed to its equilibrium value [16] and $\nu_{e e} \approx 0$, making it necessary to include collective and hydrodynamice (spatial heat flow, expansion etc.) effects, as mentioned in the Introduction, while for the treatment of collisions simpler models may be used.
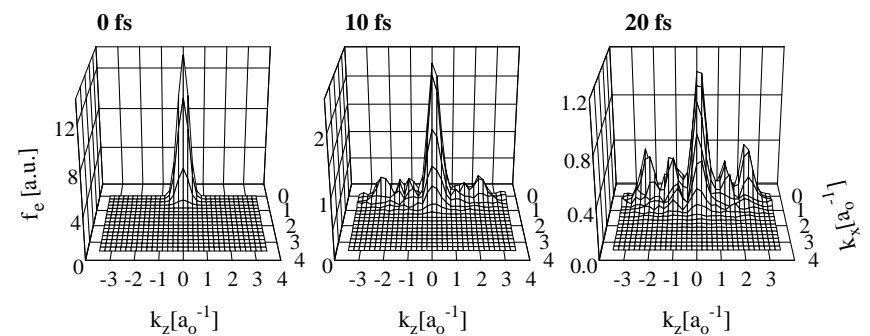

FIG. 4. Evolution of the electron distribution function in momentum space (the field is in $k_{z}$ direction). Figure shows snapshots at $t=0$ (left), after 6 laser periods (middle) and after 12 laser periods (right), when the main peak of $f_{e}$ is at the origin (the distribution as a whole oscillates with the field in $k_{z}$-direction and is isotropic in the $k_{x}-k_{y}$-plane). Note the different vertical scales.

In summary, we have presented a selfconsistent numerical investigation of the early relaxation stage, $t \leq$ $50 \mathrm{fs}$, of a pre-ionized dense plasma in a laser field with $E_{0}=10^{7} \ldots 10^{9} \mathrm{~V} / \mathrm{cm}$.. We have found the formation of a strongly nonequilibrium electron distribution giving rise to coherent bremsstrahlung emission of ultrashort third harmonics pulses. An anomalously high conversion efficiency (more than 6 orders of magnitude compared to plasmas in equilibrium) is predicted, making this fundamental process observable in experiments. The most fa- vorable conditions are expected for a pre-ionized weakly undercritical gas plasma with high charge state $\left(\nu_{e i} \sim Z\right)$ excited by a linearly polarized laser pulse with a duration $\tau_{p}$ shorter than 50fs where the third harmonics should be observable in transmission. To further improve the quantitative predictions, the plasma generation process has to be included in the simulation. For example, ionization from excited atomic levels should enhance the harmonics yield [7], accelerate the plasma heating and thus further reduce the duration of the higher harmonics pulse. Finally, we mention that our results are not limited to laser plasmas, but are equally important for electron-hole plasmas subject to intense $\mathrm{THz}$ radiation.

This work is supported by the Deutsche Forschungsgemeinschaft (Schwerpunkt "Laserfelder"), the European Commission through the TMR Network SILASI and the NIC Jülich. We acknowledge stimulating discussions with M. Schlanges and V.P. Silin.

[1] for a recent overview, see e.g. M.D. Perry, and G. Mourou, Science 264 (1994) 917

[2] M. Tabak et al., Phys. Plasmas 1 (1994) 1626

[3] Gamaliy, Laser \& Particle Beams 12, 185 (1994)

[4] W. Theobald, R. Hässner, C. Wülker, and R. Sauerbrey, Phys. Rev. Lett. 77, 298 (1996)

[5] V.P. Silin, Sov. Phys. JETP 20, 1510 (1965)

[6] The BS mechanism is fundamentally different from the collisionless harmonics generation in relativistic plasmas, see e.g. P. Gibbon, J. Quantum Electronics (1996) and refs. therein.

[7] See V.P. Silin, Sov. Phys. JETP 90, 805 (2000); Kvantovaya Elektronika (russ.) 26, 11 (1999).

[8] W. Rozmus, and V.T. Tikhonchuk, Phys. Rev. A 42, 7401 (1990)

[9] For a recent overview and further references, see D. Vick, C.E. Capjack, V. Tikhonchuk, and W. Rozmus, Comments Plasma Phys. Controlled Fusion 17, 87 (1996)

[10] H. Ruhl et al., Phys. Rev. Lett. 82, 743 (1999)

[11] A. Pukhov, and J. Meyer-ter-Vehn, Phys. Rev. Lett. 76, 3975 (1996)

[12] H. Ruhl et al., Phys. Rev. Lett. 82, 2095 (1999)

[13] J. Dawson, and C. Oberman, Phys. Fluids 5, 517 (1962)

[14] D. Kremp, Th. Bornath, M. Bonitz, and M. Schlanges, Phys. Rev. E 60, 4725 (1999).

[15] P. Hilse, to be published

[16] Th. Bornath, M. Schlanges, P. Hilse, D. Kremp, and M. Bonitz, Laser \& Particle Beams (2000) 